\title{
Duration judgment and the experience of change
}

\author{
W. DOUGLAS POYNTER and DONALD HOMA \\ Arizona State University, Tempe, Arizona
}

\begin{abstract}
Predictions based on storage size, processing effort, and change models of time estimation were tested in five experiments. The first of these presented subjects with stimulus patterns that varied on dimensions of sensory-event number and uncertainty. Subjects estimated the duration of time periods using the reproduction method. Duration estimates were most accurately predicted by the number of sensory events in each pattern. This relationship was generally positive, although the specific function relating these variables was dependent upon clock duration. The change model seemed to fit these data best. Experiment 2 demonstrated that the relationship between sensory change and judged duration was not due to the total time of sensory input. Experiments 3, 4, and 5 tested the effects of several different types of change. In Experiments 3 and 4, the number of sensory events was held constant, but the regularity of their spatial presentation was varied. In both experiments, duration judgments were positively related to the number of changes that occurred. Because the manipulations used in these experiments produced differences in the visual complexity of stimulus patterns, the argument could be made that storage size or processing effort accounted for the size of duration judgments. Experiment 5 tested the effects of change while holding the visual complexity of stimulus patterns constant. A positive relationship between duration judgments and number of changes was again found.
\end{abstract}

Given the ubiquity of man-made clocks in modern societies, it seems clear that humans frequently rely on nonorganismic mechanisms to measure the temporal extent of events. Apart from these timekeeping devices, however, we still experience something metaphorically referred to as "time passage," and can also generate estimates of duration using organismic processes. Although time perception has been the topic of scientific investigation for at least 90 years, a clear understanding of the psychological mechanisms of duration estimation is still forthcoming.

Some of the earliest researchers proposed that the experience of duration was based on the output of a biological clock (e.g., Francois, 1927; Hoagland, 1933). Recent models have emphasized the role of cognitive activity rather than metabolic functioning. This cognitive approach has the advantage of being able to explain why the quantity of stimulus information in an interval of clock time can affect its apparent duration. When increases

The manuscript is based on the first author's doctoral dissertation. Reprint requests should be addressed to $W$. Douglas Poynter, Department of Psychology, Arizona State University, Tempe, Arizona 85287. The authors would like to acknowledge the valuable contributions made by Jerry Kosisky in the performance of the experiments. They also thank Sam Leifheit and Norwood Sisson for their major contributions to the construction of the apparatus. Pat Query's patience in typing the manuscript is also greatly appreciated. Finally, comments on the manuscript by Richard Block, Dominic W. Massaro, and an anonymous reviewer were helpful and much appreciated. in stimulus information produce longer estimates of duration, this finding is sometimes referred to as the filled-duration illusion (FDI) and has been demonstrated with an impressive variety of interval "fillers," including light flashes (Buffardi, 1971), pure tone bursts (Adams, 1977; Buffardi, 1971; Thomas \& Weaver, 1975), line drawings (Mulligan \& Schiffman, 1979; Ornstein, 1969), letters and nonsense syllables (Avant, Lyman, \& Antes, 1975), and words and word lists (Block, 1974; Poynter, 1979; Thomas \& Weaver, 1975).

Generally, manipulations of the content of time intervals fall into three categories: (1) manipulations of the number of intervening events (Adams, 1977; Block, 1974; Buffardi, 1971), (2) manipulations of the complexity of stimulus events (Block, 1978; Ornstein, 1969; Schiffman and Bobko, 1974), and (3) manipulations of the amount or type of information processing required of subjects (Burnside, 1971; Hicks, Miller, \& Kinsbourne, 1976; Thomas \& Weaver, 1975).

Based on the outcome of experiments using three basic categories of experimental manipulation, three cognitive models have been developed. These will be referred to as the storage size, processing effort, and change models.' They are similar in assuming a close relationship between duration judgment and the processing of interval events; they differ in terms of the particular dimension of processing that is proposed to mediate duration experience. The storage-size notion proposes that time estimates are based on both the number and complexity of stim- 
ulus representations in memory; the processing effort model focuses on the processing time or attention requuired by interval events; and the change model emphasizes the overall amount of change experienced. The results of many experiments can be interpreted from more than one of these three perspectives, although data have usually been discussed in relation to a single model. The next section presents evidence in support of each model.

\section{Models of Duration Judgment}

Storage size model. Experiments that have tested this hypothesis often manipulate the number or memorability of items filling an interval and relate subsequent recall or recognition of these items to duration estimates. Block (1974), for example, presented subjects with 160 -sec intervals filled with 80 categorized words either blocked by category or presented in random format. The blocked condition produced significantly greater duration estimates and memory scores. The fact that memory scores paralleled apparent duration was interpreted as support for a storage size hypothesis. Poynter (1979) manipulated memory for stimulus information by filling a 195-sec interval with either 15 easy-toremember words or 15 hard-to-remember words. In addition, half of the subjects were given word prompts just prior to the time-estimation task. Subjects presented with easily recalled words and memory prompts produced the longest time estimates and the best recall performance, again demonstrating a parallel between the number of recalled stimulus events and perceived duration.

The complexity of interval stimuli has also been shown to affect duration estimates. Mulligan and Schiffman (1979) and Ornstein (1969) demonstrated that providing a simplifying code for remembering complex line drawings reduced estimates of the stimulus duration. The authors attributed this effect to a reduction in stimulus complexity. In addition, Schiffman and Bobko (1974) found that duration estimates of light-flash patterns were positively related to pattern complexity.

Processing effort model. Avant, Lyman, and Antes (1975) presented letter stimuli for very brief intervals of time $(30 \mathrm{msec})$, and found that duration estimates were inversely related to the familiarity of the stimulus. It was concluded that "the shorter apparent durations of highly familiar stimuli reflect the initiation of a more direct and automatic contact between the stimuli and some critical property of the stimuli" (Avant \& Lyman, 1975, p. 206). Such an interpretation is consistent with the idea that duration judgments are based on the time or effort required to process interval information.

Thomas and Weaver (1975), using 40- and 80msec visual presentations, found that words, and permutations of words, produced longer estimates than blank fields; in the same experiment, requiring subjects to memorize stimulus information also produced longer estimates of duration. These results were interpreted as indicating that perceived duration is positively related to processing time.

In other experiments, however, increases in processing effort have produced decreases in duration estimates. Burnside (1971) conducted an experiment in which subjects were requested to engage in processing tasks of varying difficulty (reading, adding, multiplying, etc.) during time intervals ranging from 10 to $20 \mathrm{sec}$, after which they were required to reproduce these durations. Time estimates were found to be inversely related to the difficulty of the processing task. Hicks et. al. (1976) and Vroon (1970) demonstrated that increasing the amount of active processing required of subjects reduced the apparent duration of relatively long clock intervals $(\geqslant 60 \mathrm{sec})$.

Poynter (Note 1) recently examined the effects of processing time on apparent duration. Subjects were asked to respond as rapidly as possible to questions regarding either the semantic or structural content of word stimuli. Time intervals containing these words were $11.5,18.5$, or $53.5 \mathrm{sec}$ in clock duration and contained 3,5 , or 15 words, respectively. A control condition in which subjects pressed the response button after simply reading each word was also employed.

The control group produced much larger estimates of duration than did the structural or semantic processing conditions. If it can be assumed that the reading task of the control group required less processing time than did the more complex tasks of the experimental groups (semantic or structural processing and memorization), then processing effort was negatively related to time estimates.

In summary, the relationship between duration judgments and processing effort appears to be dependent on the clock duration of the judged interval. Experiments using brief intervals (less than $100 \mathrm{msec}$ in duration) demonstrate a positive relationship (Avant et al., 1975; Thomas \& Weaver, 1975); the majority of experiments using longer clock durations (greater than $10 \mathrm{sec}$ ) have shown the inverse relationship (Burnside, 1971; Hicks et al., 1976; Vroon, 1970; Poynter, Note 1).

Change model. The idea that psychological duration is based on the experience of change is commonly associated with Fraisse (1963). Recently, Block and Reed (1978) revived this notion by proposing that duration estimates are based on "memory for the overall amount of change in cognitive context (experienced) during an interval' (Block \& Reed, 1978 , p. 656). This idea appears particularly promising in light of the fact that recent experimental findings that support this change hypothesis are 
difficult to explain using either storage size or processing effort models. In Block's experiment, subjects were requested to process a sequentially presented list of words filling a $160-\mathrm{sec}$ time interval. The way in which these words were processed varied between three conditions. In one condition, all words were semantically processed. A second condition required structural analysis (identifying upper- or lowercase letter form). The third condition required subjects to alternate between semantic and structural processing. Duration estimates of the time interval during which these forms of processing took place were longest for the alternating condition, even though "storage size" (as measured by recognition scores for list items) was greater in the single-semantic processing condition; processing effort does not appear to account for these results either, since the single-semantic processing task should also have required the most processing time (see Craik \& Tulving, 1975). Block proposed that duration estimates were longer when alternating tasks were performed because the number of changes in "process context" was greater in this condition.

In two experiments, Poynter (1983) presented subjects with 170 - and 225 -sec time periods filled with word lists that contained high-priority events clustered at either the beginning or the end of the list (unsegmented condition) or dispersed evenly throughout (segmented condition). In both experiments, subjects presented with the segmented word lists produced longer estimates than those presented with the unsegmented lists. Because this effect was produced without affecting recall or recognition performance for word-list items, results were attributed to the number of segments into which intervals were divided. Because changes delimited interval segments, this result further supports the change model.

\section{EXPERIMENT 1}

Experiment 1 was an exploratory study designed to compare the predictive utility of the storage size, processing effort, and change models of time perception. A paradigm was employed which required subjects to either observe or memorize a stimulus pattern, then estimate its duration and, in some cases, recall the pattern of the events that were presented. Stimulus patterns were sequences of light flashes presented in various spatial configurations. These patterns varied on dimensions of sensory event number, uncertainty, and clock duration.

Half of the subjects were asked to memorize stimulus patterns (high-processing effort condition), and the other half were asked to simply observe them (the low-processing effort condition). While memorizing stimuli would be expected to require more processing time than simple observation (cf. Thomas \& Weaver, 1975), it might also be exptected to expand storage size. A second manipulation of processing effort (pattern uncertainty) was therefore employed. This variable would not be expected to produce parallel effects on storage size. Recall accuracy of stimulus patterns was measured as a dependent variable and provided a check on storage size. The number of sensory events in the interval served as a measure of the amount of change experienced. The number of light flashes presented during time intervals varied both between and within the two stimulus sets employed.

The experiment was designed to determine whether time estimates covaried with levels of processing, sensory-event number, pattern uncertainty, and/or recall accuracy. Results were compared with the predictions made by storage size, processing effort, and change models of time estimation.

\section{Method}

Subjects. Seventeen students enrolled in introductory psychology courses at Arizona State University were subjects in Experiment 1. The students were given class credit for their participation.

Stimuli. Light flashes were chosen as the basic stimulus unit. The complexity of stimulus information was manipulated by varying the regularity of the spatial pattern formed by these light flashes. In this way, complexity could be varied without changing the number of events processed. In the interpretation of previous studies (Burnside, 1971, for example), it is sometimes unclear whether performing a small number of complex tasks (multiplying numbers, for example) required more processing effort than performing a large number of simple tasks (reading digits, for example).

Two stimulus sets were generated. These were called Complement Set $\mathbf{A}$ and Complement Set B, and they are illustrated in Figure 1. Each set contained 10 patterns, which differed in terms of the number and arrangement of light flashes that occurred along a horizontal path. Light flashes were 1-in.-diam circles and were blue in color; they were always presented in left-toright order. The stimulus patterns were chosen in order to instantiate as broad a range of recall accuracy and number of sensory events as possible. While there was no accurate way of predicting recall accuracy for potential patterns, some guidance in this regard was provided by a previous experiment (Glanzer \& Clark, 1963), in which recall accuracy for binary-event patterns was measured.

Because subjects were told that light flashes could occur at any one or at all of eight different spatial locations, it was assumed that each pattern could be defined as containing eight binary events. Events had two possible outcome categoriesa spatial location could be either lit or not lit. For each pattern, the average uncertainty of pairs of events was calculated using Shannon's uncertainty measure (Garner, 1975, p. 21). The categories of event pairs were on-on, on-off, off-on, and off-off. As illustrated in Figure 1, patterns in B were complements of patterns in $\mathbf{A}$, and therefore had identical values on the uncertainty measure.

Five different interval durations were used in this experiment$.8,2.4,4.0,6.4$, and $16.0 \mathrm{sec}$. These interval lengths correspond to individual event durations of $.1, .3, .5, .8$, and $2.0 \mathrm{sec}$. Memory performance was measured at each of these five clock duration levels. Four groups of subjects were used: two groups were shown Complement A patterns, and the other two groups 


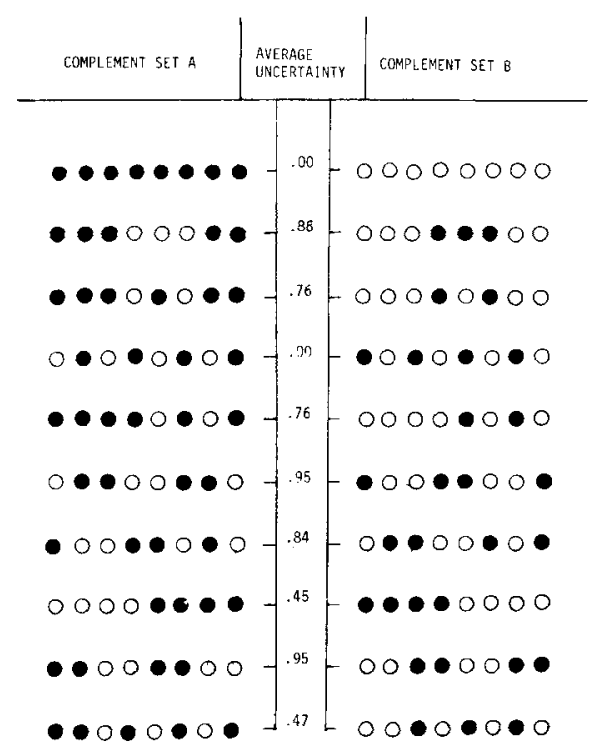

Figure 1. Stimulus patterns presented in Complement Sets A and $B$, with average uncertainty values for each pattern.

saw Complement B patterns; subjects in one of the two subgroups were instructed to memorize the patterns, while those of the other subgroup were instructed to simply observe them. Each of the 10 patterns in both sets was presented at each of the five clock durations, making a total of 50 intervals experienced by each subject. Each subject saw a different randomization of the 50 intervals.

An onset light flashed for $1 \mathrm{sec}$ prior to the start of each interval to signal its beginning, and an offset light flashed immediately after the last event of the interval to signify its end.

Design. A $2 \times 2 \times 10 \times 5$ design was used, with two betweenand two within-group factors. The between-group factors were complement set (A and B) and processing task (memory and nomemory). The within-group factors were stimulus pattern (1, $2,3, \ldots, 9,10)$, and clock duration $(.8,2.4,4.0,6.4$, and $16 \mathrm{sec})$. Duration estimation (measured as reproduced time in seconds) was the primary dependent variable. Although recall accuracy of the stimulus patterns was technically a dependent measure, it was used as a predictor variable to evaluate duration estimation data. Two other variables were also used to evaluate duration estimates: (1) number of light flashes in stimulus patterns, and (2) average uncertainty of stimulus patterns.

Procedure. All subjects were informed that the experiment concerned time perception; in addition, subjects in the memory condition were told that memory for event sequences was also being studied. Each subject was seated in a chair positioned directly in front of the light board at a distance of approximately $5 \mathrm{~m}$. The location of each event was described by pointing to the position on the light board where it would appear. It was further explained that 50 eight-event sequences would be presented consecutively, and that an event would consist of a position's being either on or off. The positions of the onset and offset lights were demonstrated, and their purpose was described as indicating the beginning and end of an interval, respectively. In the no-memory condition, the subjects were told that following the offset light of each of the 50 intervals, they would be asked to reproduce that interval's duration by depressing the response key twice, the first time to start a timer and the second time to stop it. In the memory condition, the subjects were told that, in addition to the time estimation task, they would be required to recall the sequence of events. This task followed the time-reproduction task; the subjects responded by reproducing the sequence on a prepared form which represented the spatial locations of events with eight blank circles scaled to match the dimensions of the original sequences.

\section{Results}

Each of the four factors had significant effects on duration estimates. Reproduced duration was a positive function of clock duration $[F(4,52)=331.6$, $\mathrm{p}<.001]$. Figure 2 represents the relationship between reproduced duration and clock duration for Complement Sets A and B. As this figure demonstrates, the stimulus set experienced (A vs. B) also affected estimated duration $[\mathrm{F}(1,13)=5.31, \mathrm{p}<.05]$; Complement A patterns were judged as being longer than Complement $B$ patterns. The magnitude of this effect increased with increasing clock duration, accounting for a complement set $\times$ clock duration interaction $[F(4,52)=4.57, p<.01]$. As will be discussed later, the main effect of complement set is consistent with differences in the average number of light flashes that occurred in these sets (an average of five in A and three in B).

The stimulus pattern factor also had a main effect $[F(9,117)=4.01, p<.001]$. Supplementary trend analyses were performed in order to determine which of three variables previously mentioned (recall accuracy, average uncertainty, or number of light flashes) best described the pattern of duration estimates accounting for this effect. Because the interaction between stimulus pattern and complement set was significant $[F(9,117)=3.77, p<.001]$, data for Complement Sets A and B were analyzed separately. Table 1 shows mean duration estimates ordered according to stimulus-pattern uncertainty and recall accuracy. Neither of these orderings produced significant linear or quadratic trends for both

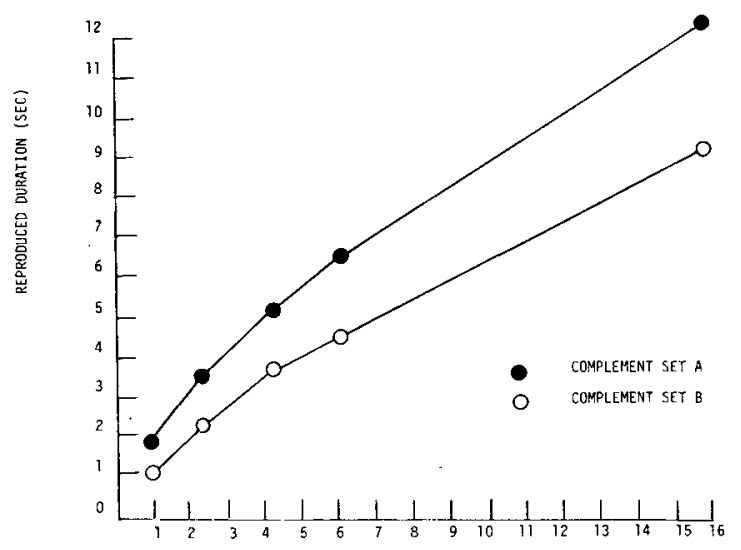

CLOCK DURATION (SEC)

Figure 2. Reproduced duration as a function of clock duration for Complement Sets $A$ and $B$. 
Table 1

Reproduced Duration for Complement Sets A and B, Ordered by Stimulus-Pattern Uncertainty and Recall Accuracy

\begin{tabular}{|c|c|c|}
\hline & \multicolumn{2}{|c|}{ Reproduced Duration (sec) } \\
\hline & $\begin{array}{l}\text { Complement } \\
\text { Set A }\end{array}$ & $\begin{array}{c}\text { Complement } \\
\text { Set B }\end{array}$ \\
\hline \multicolumn{3}{|c|}{ Average Uncertainty } \\
\hline $\begin{array}{r}.00 \\
.00 \\
.45 \\
.47 \\
.76 \\
.76 \\
.84 \\
.86 \\
.95 \\
.95\end{array}$ & $\begin{array}{l}6.49 \\
5.62 \\
5.58 \\
5.89 \\
5.59 \\
5.58 \\
5.49 \\
6.02 \\
5.84 \\
5.54\end{array}$ & $\begin{array}{l}5.23 \\
4.76 \\
5.26 \\
4.28 \\
4.00 \\
4.60 \\
3.97 \\
4.11 \\
3.91 \\
4.40\end{array}$ \\
\hline \multicolumn{3}{|c|}{ Average Recall Accuracy } \\
\hline $\begin{array}{l}6.3 \\
6.6 \\
6.7 \\
6.8 \\
6.9 \\
6.9 \\
7.0 \\
7.7 \\
7.7 \\
7.9\end{array}$ & $\begin{array}{l}5.89 \\
5.59 \\
5.49 \\
5.54 \\
5.84 \\
5.58 \\
5.62 \\
5.58 \\
6.02 \\
6.49\end{array}$ & $\begin{array}{l}4.28 \\
4.00 \\
3.97 \\
4.40 \\
3.91 \\
4.60 \\
4.76 \\
5.26 \\
4.11 \\
5.23\end{array}$ \\
\hline
\end{tabular}

Table 2

Mean Duration Estimates (Seconds) as a Function of the Number of Light Flashes Presented

\begin{tabular}{|c|c|c|c|c|c|c|c|}
\hline \multirow{2}{*}{$\begin{array}{c}\text { Clock } \\
\text { Duration }\end{array}$} & \multicolumn{7}{|c|}{ Number of Light Flashes } \\
\hline & 0 & 2 & 3 & 4 & 5 & 6 & 8 \\
\hline & \multicolumn{7}{|c|}{ Complement Set A } \\
\hline & & & & 5.57 & 6.08 & 5.80 & 6.48 \\
\hline & \multicolumn{7}{|c|}{ Complement Set B } \\
\hline $\begin{array}{r}.8 \\
2.4 \\
4.0 \\
6.4 \\
16.0\end{array}$ & $\begin{array}{r}.99 \\
2.12 \\
4.17 \\
6.07 \\
12.22\end{array}$ & $\begin{array}{l}1.29 \\
2.71 \\
3.53 \\
4.53 \\
9.62\end{array}$ & $\begin{array}{l}1.47 \\
2.69 \\
3.38 \\
4.66 \\
8.88\end{array}$ & $\begin{array}{l}1.31 \\
2.83 \\
4.04 \\
5.13 \\
9.18\end{array}$ & & & \\
\hline
\end{tabular}

Note-The effect of light flashes depended on clock duration in Complement Set $B$; data for the set are therefore tabled by levels of duration, which are indicated in column 1.

stimulus sets. Only one variable, number of light flashes, demonstrated a significant trend with duration estimates for both sets of stimulus patterns. For Complement Set $\mathrm{A}$, the trend was positive and linear $[F(1,7)=14.19, p<.01]$. Table 2 presents mean estimates tabled by number of light flashes for Complement Sets A and B. In Set B, the function relating these two variables changed across the fiye clock durations used in the experiment, producing a significant clock duration $\times$ stimulus pattern interaction $[F(36,216)=29.71, p<.001]$. For the briefest durations $(.8$ and $2.4 \mathrm{sec})$, the trend appeared to be positive and linear. For the longer durations, it was $\mathrm{U}$ shaped. Figure 3 illustrates those trends.

Although no main effect of processing task was found, a processing task $\times$ clock duration interaction was significant $[\mathrm{F}(4,52)=5.58, \mathrm{p}<.001]$. Figure 4 illustrates this apparent "crossover" interaction. It resulted from duration estimates' being larger for groups required to memorize stimulus patterns for the .8- and 2.4-sec clock intervals and larger for groups required only to observe them for the 6.4- and 16.0-sec intervals.

\section{Discussion}

The variable most consistently related to duration estimates was sensory change. The finding that stimulus patterns in Complement Set $A$ were estimated to be longer than those in B was interpreted as support for the change model, because the average number of light flashes was greater in $A$ than in $B$ (an average of five in $A$ and three in B). The mean of recall scores for patterns in Set $A$ was not significantly different from the mean recall score for patterns of Set B. If the accuracy of pattern recall reflects the amount of information retained in memory, then the storage size model cannot explain the longer duration estimates associated with set $\mathbf{A}$ patterns. The 10 stimulus patterns in $B$ were also equal to the complementary patterns in $A$ on the dimension of stimulus uncertainty. If the amount

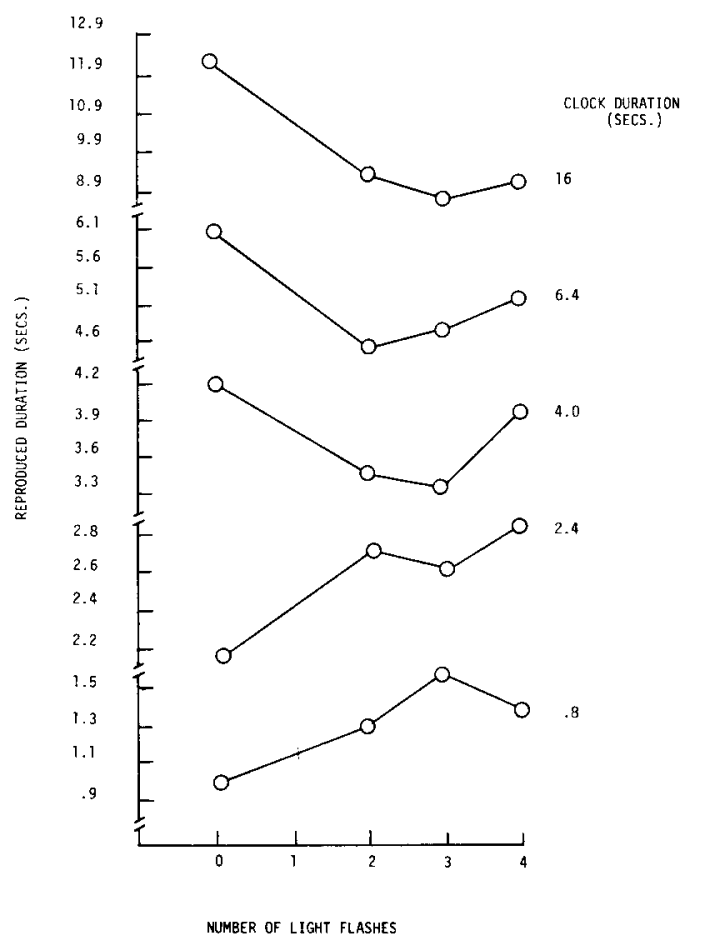

Figure 3. Reproduced duration as a function of number of light flashes for Complement Set B. 


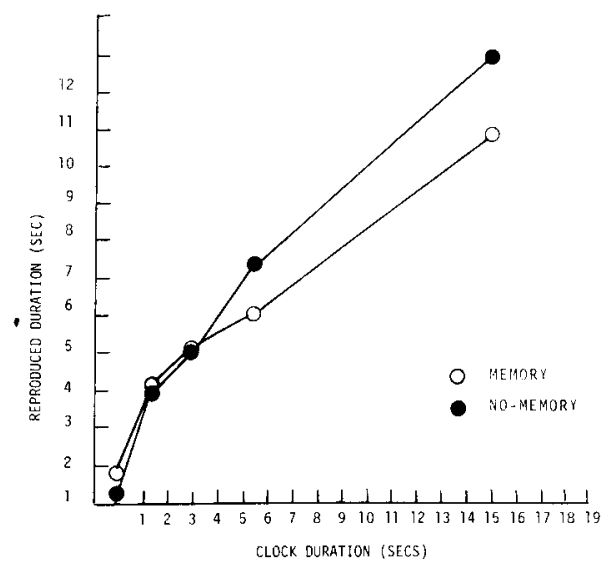

Figure 4. Reproduced duration as a function of clock duration for the two levels of the processing-task factor.

of processing required of stimulus patterns can be accurately represented by the measure of uncertainty used here, then a model based on processing effort would not predict the observed difference in duration estimates either.

Within each set of stimulus patterns, reproduced duration was also found to be most closely related to the number of sensory changes that occurred. It is important to note that the relationship between these variables was dependent upon the clock duration of the interval being judged. For short durations ( $<$ a few seconds), more changes produced longer estimates. For longer durations, intervals without stimulus changes were judged to be longer than intervals filled with them. Apparently, the intuitive notion of "filled" time passing more rapidly than "empty" time is true only when the ratio of filler events to clock time falls below a certain limit.

This conclusion is also supported by the interaction between processing task and clock duration (see Figure 4). Requiring memorization of stimulus patterns expanded perceived time for intervals less than a few seconds and contracted it for longer intervals. This finding helps to reconcile previous experiments demonstrating positive and negative relationships between amount of processing and judged duration. It could also be interpreted as replicating the interaction described in the previous paragraph between number of changes and clock duration. For brief intervals, requiring memorization of stimulus patterns presumably stimulated subjects to attend more assiduously to the number and location of events occurring in the interval, leading to an increase in the number of changes logged in memory and a subsequent lengthening of judged duration; this parallels the increase in duration estimates observed when the actual number of events presented was increased in brief intervals. For longer durations, this heightened attention to stimulus events produced shorter estimates, as observed when the actual number of events presented was increased.

If change is the unit of time upon which psychological duration is based, then changes unrelated to overt sensory events accounted for the duration estimates of long, empty intervals. The source of this form of change must be organismic. If this assumption is accepted, Experiment 1 has demonstrated that, for relatively long clock durations, attending to a small number of sensory changes nets fewer units of psychological time than attending to organismic change. This finding is consistent with the fact that some forms of organismic change occur cyclically as part of biological maintenance (e.g., heart beats and breaths). The term sensory change is used here to refer to discrete afferent impulse in response to exteroceptive stimuli (light flashes, auditory pulses, etc.). Organismic change refers to afferent impulse in response to proprioceptive stimuli (relative position of body segments, position of body in space) or interoceptive stimuli (e.g., glucose levels, blood pressure), and also to cognitive events that are not the direct result of sensory receptor stimulation (including neural events associated with "shifts of attention," changes from one step to another in information processing sequences, or perhaps changes in locality of neural-space excitation).

While the distinction between these categories can become arbitrary, it is convenient to use them in reference to previous experiments that manipulate number or complexity of exteroceptive stimuli (analogous to "sensory changes") or amount and type of information processing (analogous to "cognitive-organismic changes"'). The terms sensory and organismic are therefore useful in the analysis of previous findings.

Experiment 1 has demonstrated the effect of sensory change on duration estimates; Experiments 3, 4 , and 5 will test the effects of cognitive change.

\section{EXPERIMENT 2}

The results of Experiment 1 are consistent with previous studies which have shown that increasing the number of tone bursts in a time interval increases its perceived duration (Adams, 1977; Buffardi, 1971). In both Experiment 1 and these past studies, however, the number of events presented was perfectly confounded with the total time of sensory input.

Experiment 2 presented subjects with $.8 \mathrm{sec}$ of light divided into 1,4 , or 8 discrete sensory parcels. In order to control for the effects of spatial arrangement, all intervening light flashes were presented in the same spatial location. 
The change model predicted that apparent duration would be related to the number of discrete pulses into which the sensory stimulation was divided.

\section{Method}

Subjects. Eighteen introductory psychology students participated in the experiment as partial fulfillment of class requirements.

Apparatus, Stimulus materials, and Design. The apparatus used in Experiment 1 was also employed in this experiment. The laboratory room remained dimly lit by a small, shaded desk lamp through all experimental sessions.

All time intervals were $1.8 \mathrm{sec}$ in duration. Sensory input was presented at one spatial location on the light board apparatus. As in Experiment 1, light pulses were circular in shape and 1 in. in diameter; the lights were blue in color. The duration of light stimulation was held constant at $.8 \mathrm{sec}$, but the number of pulses used to present this stimulus duration varied between conditions. Figure 5 illustrates the event sequences presented in Experiment 2.

In the one-pulse condition, the $.8 \mathrm{sec}$ of light was presented continuously from the beginning of the interval, leaving $1.0 \mathrm{sec}$ of empty time at the end. In the four-pulse condition, .2-sec pulses were presented every $.3 \mathrm{sec}$; in the eight-pulse condition, eight .1 -sec pulses were presented every $.143 \mathrm{sec}$. A 1 -in.-diam red onset light, whose duration was $1 \mathrm{sec}$, signaled the beginning of an interval, and a 2 -in. blue light signaled its end. The offset light remained lit until the next interval was electronically initiated by the experimenter, who sat several meters behind subjects.

Duration judgments were measured in a forced-choice discrimination task. Although the argument can be made that employing such a method may force subjects to attend to nontemporal information in order to make their duration judgments (cf. Thomas \& Brown, 1974), employing the reproduction method for brief durations requires consideration of limits on muscular coordination and response time. The forced-choice method was justified in the present experiment because, in at least two previous FDI studies that used intervening stimuli and clock durations similar to those used here, the same effect of stimulus number on perceived duration was demonstrated with reproduction and discrimination tasks (Buffardi, 1971; Thomas \& Brown, 1974). The use of a forced-choice discrimination task was also supported by pilot data suggesting (1) that subjects found the task straightforward and (2) that it would result in time estimates of low variance.

The three experimental intervals presented in Figure 5 and an additional filler interval were presented in all possible pairwise combinations (including $\mathbf{A B}$ and $\mathrm{BA}$ orders), making a total of 12 pairs presented. The filler interval contained a sequence of eight .1-sec pulses distributed in left-to-right order across a hori-

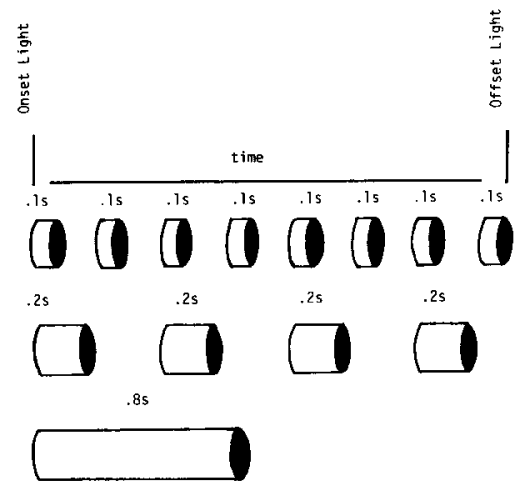

Figure 5. Stimulus patterns presented in each of the three conditions of Experiment 2. zontal distance of $7 \mathrm{ft}$. This filler was added to expand the number and complexity of pairwise combinations presented. It was assumed that this would reduce the chance of subjects' making artificial duration judgments based on a single, outstanding stimulus dimension.

The second member of an interval pair was presented approximately 1 to $2 \mathrm{sec}$ after the first. The clock time between interval pairs varied, but generally was close to $5 \mathrm{sec}$, the delay being determined by the speed with which subjects responded to the implied question, "Which interval was longer, the first or the second?" Two randomizations of interval pairs served to establish presentation order. Data from these two orders were combined for analysis.

Procedure. Each subject was informed that the experiment concerned the perception of light sequences. Prior to the experimental sessions, the subjects were shown the locations of onset and offset lights and familiarized with their meaning. The spatial location of light-pulse presentations was expressed by hand gesture. The subjects were told that they would experience a number of time intervals presented in pairs, and that each interval of a pair would be bounded by the red onset light and large blue offset light. The subjects were informed of the size and color of light pulses filling the experimental intervals. Following the second member of a pair, the subjects were to mentally compare the duration of the two intervals and respond by writing on a response sheet the number " 1 " or the number " 2 ," depending on which of the two intervals they felt was longer. Subjects were asked to look up from their response sheets after completing this task in order to signal the experimenter that they were ready to view the next pair of intervals. Final instructions requested that tapping or counting strategies not be used to remember the duration of intervals. The experimenter triggered the onset light by depressing a control key when the subjects were ready for the first and all subsequent pairs of sequences.

\section{Results}

The number of times that each of the three intervals (one-pulse, four-pulse, and eight-pulse) was chosen as being longer in duration than the interval with which it was paired served as the dependent measure. This variable will be referred to as frequency of choice (FOC) and is assumed to reflect the perceived duration of time intervals. Since each of the three experimental intervals was paired twice with the other two, and twice with the filler interval, the maximum FOC score was 6.

Figure 6 represents the relationship between FOC and the number of light pulses in the interval. ${ }^{2}$ A oneway ANOVA performed on these data indicated a significant main effect $[F(2,34)=6.85, p<.01]$, the linear component of which was also significant $[F(1,17)$ $=17.58, \mathrm{p}<.001]$. This result indicated that duration judgments in Experiment 2 were a positive linear function of the number of light pulses presented.

\section{Discussion}

Previous studies (e.g., Buffardi, 1971; Thomas \& Brown, 1974) which have tested the effects of stimulus number on perceived duration have confounded this independent variable with the total time of sensory input. Experiment 2 has demonstrated the effects of light-pulse number in the absence of differ- 


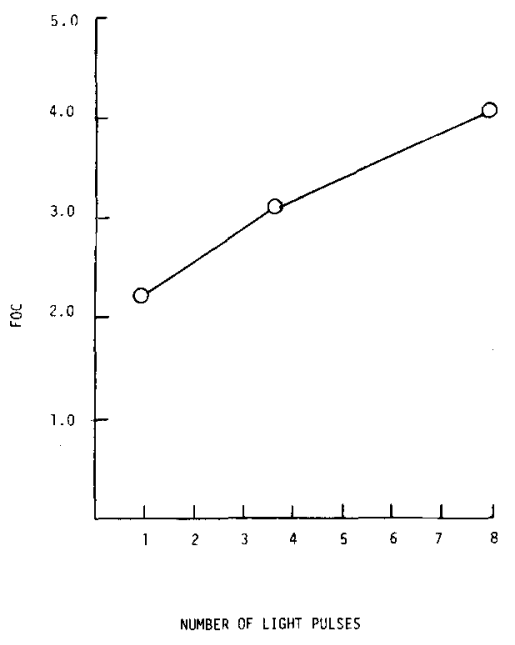

Figure 6. Duration judgment (FOC) as a function of the number of light pulses.

ences in sensory input time. These results provide additional support for a model of time perception based on change.

In the discussion of Experiment 1, it was proposed that the interaction between clock duration and stimulus number could be explained by considering the effects or organismic change on perceived duration.

Block and Reed (1978) demonstrated that increasing the number of changes in processing context (i.e., the type of cognitive analysis required) produced increases in duration estimates. In their experiment, linguistic stimuli were processed on either a structural or a semantic level. Alternating between these two types of processing was assumed to produce more cognitive change than performing only one type of processing.

The following experiment uses a less complex stimulus unit to manipulate organismic-cognitive change.

\section{EXPERIMENT 3}

In several previous studies, the effect of stimulus complexity on duration judgments could also be attributed to the amount of cognitive change experienced. Block (1978), for example, independently varied the complexity of individual stimulus items (black and white bar patterns) and the complexity of sequences of these stimulus items. While no effect of individual-item complexity was found, duration estimates were found to increase with increasing complexity of stimulus sequences. Block interpreted these results as supporting the hypothesis that duration estimates were constructed from the amount of change experienced in cognitive context.

A change model could also be used to explain the results of other studies. In the Schiffman and Bobko experiment, three stimulus displays were employed; each consisted of two rows of four lights. In the "low-complexity" condition, these lights came on simultaneously and stayed on for the entire interval. In the "intermediate-complexity" condition, all lights flashed on and off simultaneously at a rate of two flashes per second. Finally, in the "highcomplexity" condition, lights flashed on and off at approximately the same rate, but not simultaneously. The more broken flash patterns of the high-complexity condition produced much longer duration estimates than the intermediate and low-complexity conditions. This result is consistent with predictions of a change model, since many more changes in the spatial relationships between light flashes were produced in the high-complexity display.

Experiment 3 also employed light flashes as the basic stimulus unit. The amount of cognitive change was manipulated by varying the distance between adjacent light flashes presented sequentially in leftto-right order across a horizontal field ranging from 5 to $11 \mathrm{ft}$ in length. The number of sensory events presented was held constant across conditions.

\section{Method}

Subjects. Sixteen introductory psychology students participated in the experiment as partial fulfillment of class requirements.

Design. Experiment 3 used a one-factor, repeated measures design. The trial factor, changes in spatial separation, had four levels $(0,1,2,3)$. FOC, a reflection of perceived duration, was again used as the dependent measure.

Apparatus, Stimulus materials, and Procedure. The apparatus and procedure were the same as those used in Experiment 2 . The duration of all intervals was $1.1 \mathrm{sec}$; the duration of each light flash and the interflash duration were both $.1 \mathrm{sec}$. Six light flashes were presented during each interval. Figure 7 illustrates the visual patterns presented during each of the four trial conditions. A filler interval was again added to expand the number of pairwise combinations presented to subjects. The total number of combinations (including AB and BA order) was 20. The warning lights used to delimit the intervals were the same as those described in Experiments 1 and 2. As in Experiment 2, a forced-choice discrimination task was used to elicit time judgments.

\section{Results}

Because each visual pattern used in the experiment was presented eight times, the maximum FOC score

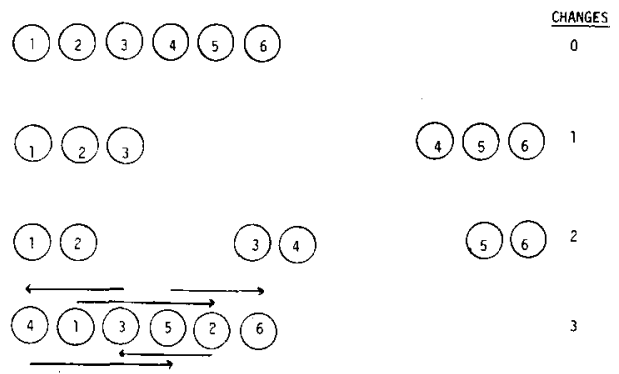

Figure 7. Stimulus patterns used in Experiment 3. Arrows illustrate the direction of presentation; numbers inside circles indicate order of presentation. 
was 8. Number of changes had a significant effect on judged duration $[F(3,45)=3.98, p<.05]$. The linear component of this effect was also significant $[F(1,15)$ $=12.92, \mathrm{p}<.01]$. This relationship is represented in Figure 8. ${ }^{3}$

\section{Discussion}

These results establish that changes other than those related to the absolute number of sensory events contribute to the experience of duration. Unfortunately, the present manipulation of cognitive change produced stimulus patterns which also differed in terms of the length of the stimulus field (this varied from 5 to $11 \mathrm{ft}$ ) and the spatial locations where light flashes occurred. It is possible that these uncontrolled differences in the physical characteristics of stimulus patterns may have affected duration estimates in an unknown way. Experiment 4 employed a manipulation of change that controlled these unwanted differences.

\section{EXPERIMENT 4}

Experiment 4 used stimulus patterns that were identical in (1) the number of sensory events presented, (2) the length of the stimulus field, and (3) the specific locations where light flashes occurred. The order in which these locations were lit was varied to manipulate the amount of cognitive change experienced. The independent variable was the number of direction changes that occurred during presentation of each stimulus pattern.

Duration judgments were predicted to vary with the number of direction changes that occurred.

\section{Method}

Subjects. Eighteen introductory psychology students participated for class credit as subjects in the experiment.

Stimulus materials. The stimulus patterns used in this experiment are shown in Figure 9. Each of the eight flashes and all interflash intervals were .1 sec in duration, making the total duration

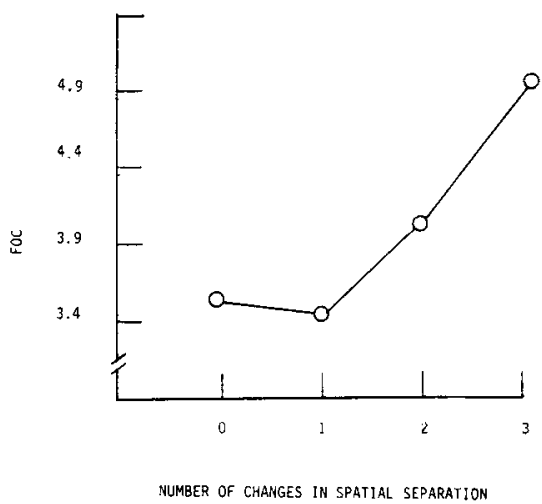

Figure 8. Duration judgment (FOC) as a function of the number of changes in spatial separation.

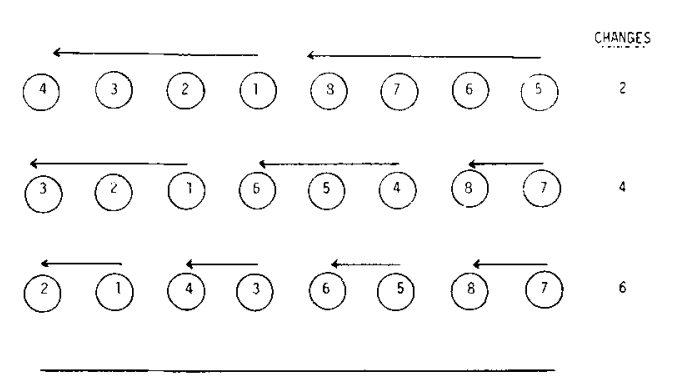

Figure 9. Stimulus patterns used in Experiment 4. Arrows indicate direction of presentation; numbers within circles indicate order of presentation.

$1.5 \mathrm{sec}$ for all intervals. Again, a filler interval was employed, making the total number of combinations presented equal to 12 .

Design, Apparatus, and Procedure. The number of direction changes served as the independent variable and had three levels $(2,4$, and 6$)$. The dependent variable was again FOC, the number of times that each visual pattern was chosen as being longer in duration than the patterns with which it was paired.

The apparatus and procedure were identical to those in Experiment 3.

\section{Results}

Because each stimulus pattern was presented six times, the maximum FOC score was 6 . The trial factor, number of direction changes, produced a significant effect on $F O C[F(2,34)=8.64, p<.001]$. The linear component of this effect was also significant $[F(1,17)=12.49, \mathrm{p}<.01]$. As in Experiment 3, these results demonstrated a linear relationship between duration judgments and the number of cognitive changes experienced. Figure 10 illustrates this relationship. ${ }^{4}$

\section{Discussion}

One criticism of the manipulations used in Experiments 3 and 4 is that, in addition to producing differences in the number of cognitive changes experienced, they also produced differences in the vi-

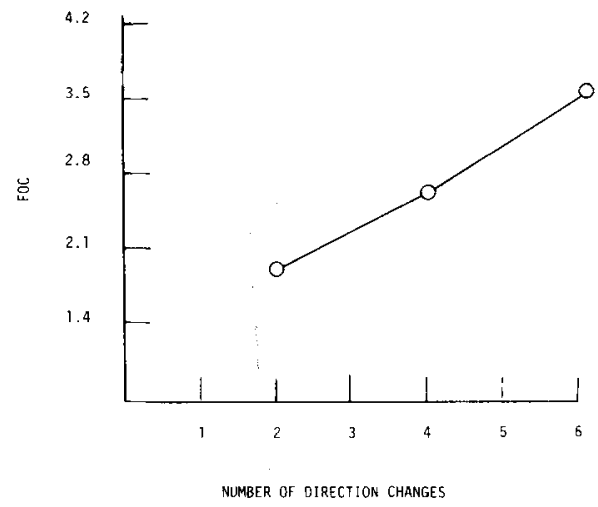

Figure 10. Duration judgment (FOC) as a function of the number of direction changes. 
sual appearance of the stimulus arrays. As a result, it could be argued that patterns with a large number of cognitive changes were also high in visual complexity. The results of Experiments 3 and 4 could then be explained using either storage size or processing effort models, since both storage size and processing effort should increase with increasing stimulus complexity. To establish the effects of cognitive change, a manipulation that controlled differences in the visual complexity of stimulus patterns was needed.

\section{EXPERIMENT 5}

In Experiment 5, all intervals contained exactly the same visual information. The total "tracking" distance, the number and location of light flashes, the distance between adjacent light flashes, and the presentation order of light flashes were identical in all stimulus patterns. Cognitive change was manipulated by varying the temporal distance between light flashes. The factors contributing to change in this experiment were assumed to be strictly cognitive, since the spatial characteristics of stimulus patterns were identical.

\section{Method}

Subjects. Thirteen introductory psychology students participated in the experiment in partial fulfillment of class requirements.

Stimulus materials. The total duration of all intervals was $2.1 \mathrm{sec}$. As in the previous two experiments, light flashes were $.1 \mathrm{sec}$ in duration. The size and variability of the interstimulus duration was dependent upon the level of the independent variable. Figure 11 illustrates the stimulus patterns employed in this experiment. These patterns, together with two filler intervals, were presented in all possible pairwise combinations, making a total of 20 pairs presented.

Design, Apparatus, and Procedure. Number of cognitive changes was the independent variable and had three levels $(0,2$, and 4). The dependent variable was FOC. The apparatus and procedure were the same as those used in Experiments 3 and 4.

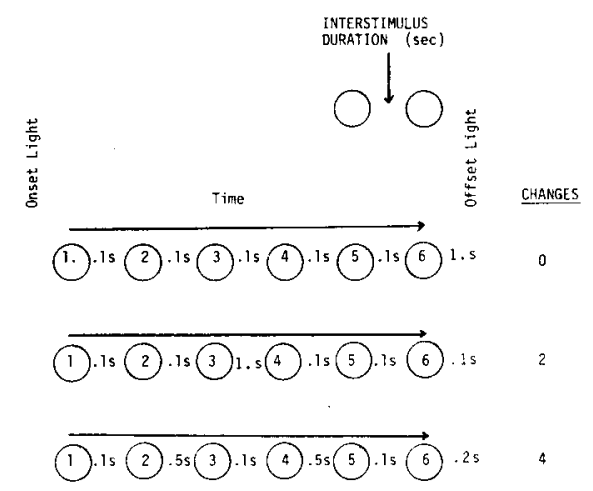

Figure 11. Stimulus patterns used in Experiment 5. Arrows indicate direction of presentation; numbers within circles indicate order of presentation.

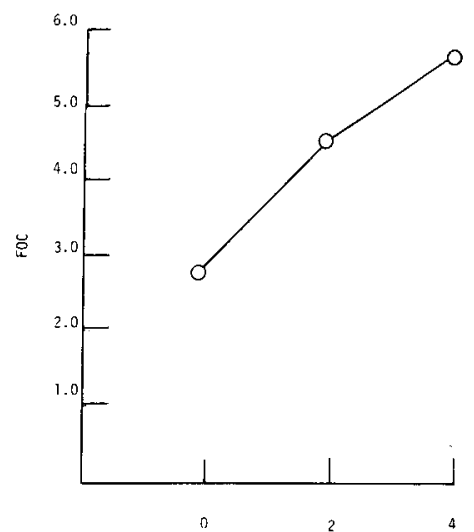

NUMBER OF CHANGES IN INTERSTIMULUS DURATION

Figure 12. Duration judgment (FOC) as a function of the number of interstimulus duration changes.

\section{Results}

Because each stimulus pattern was presented eight times, the maximum FOC score was 8. The trial factor produced a significant effect on duration estimates $[F(2,24)=8.87, p<.001]$; the linear component of this effect was also significant $[F(1,12)=$ $25.19, \mathrm{p}<.001]$. These results demonstrated a positive linear relationship between judged duration and the number of cognitive changes experienced. Figure 12 illustrates this relationship. ${ }^{5}$

\section{Discussion}

Experiment 5 demonstrated that changes unrelated to spatial complexity can affect estimates of duration. Duration judgments were positively related to the number of changes in temporal separation of light flashes, despite the fact that the spatial characteristics of the stimulus patterns were identical. For reasons discussed below, explanations of this finding based on processing effort or storage size do not seem appropriate.

According to the storage size model, psychological time is based on the number and complexity of encoded memories. In the present experiment, variability of subinterval durations would have to account for any differences in complexity; but in order for subjects to detect this subinterval variability, they would first have to "measure" or time subinterval durations. This is a paradox, because the time percept is antecedent to its precursor (in this experiment, temporal complexity).

Because the processing effort model also relies on the construct of temporal complexity to explain the present finding, it faces the same logical difficulty. It is assumed that temporal complexity affects the cognitive effort required to process interval informa- 
tion, which in turn determines the size of duration estimates; but, in order to be sensitive to temporal complexity, detection of differences in subinterval durations is prerequisite.

Proposing the existence of an underlying timer whose output affects the way in which information is processed and/or stored would resolve the difficulty these two models encounter in explaining the results of Experiment 5.

A model based on change must also account for the detection of differences in subinterval duration, since the variability of these durations was used as a measure of the amount of cognitive change experienced. Changes in subinterval duration could be detected by counting units of organismic change (like interoceptive, proprioceptive, or apperceptive events), then comparing the sum of these units for each subinterval. This explanation has the advantage of not relying on the existence of a timing mechanism that is independent of change. The other two models must account for these data by assuming a timing mechanism independent of storage size and processing effort. On this basis, the change model seems most parsimonious.

\section{GENERAL DISCUSSION}

Many studies have demonstrated the effects of stimulus information on duration judgments. In some cases, the effect is a lengthening of perceived time; in others, it is a shortening. A review of the literature suggested actual clock duration of the judged interval might determine the direction of this temporal distortion. Experiment 1 provided empirical evidence of this apparent interaction between clock duration and stimulus information. Figure 13 presents a graphical representation of this relationship, the stippled portion being a composite of data represented in Figures 3 and 4.

Experiment 1 also tested the predictive utility of three cognitive models of duration judgment. One

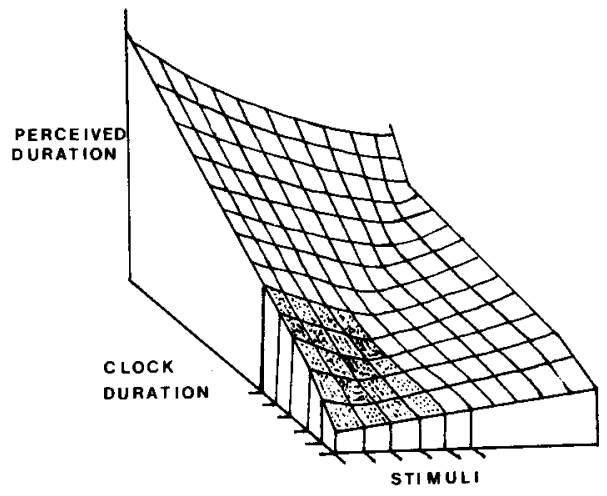

Figure 13. Duration judgment as a function of both number of stimuli (or amount of stimulus processing) and clock duration. emphasizes number and complexity of accessible memories, another the amount of processing that occurs, and the third, the amount of change experienced. Number of changes (as measured by number of sensory events) was the best predictor of duration estimates.

The argument could be made that stimulus-pattern uncertainty proved ineffectual as a predictor of duration estimates because it did not adequately reflect the cognitive effort required to process stimulus patterns. It was assumed that "light on" and "light off" could be used as outcome categories equal in information content; the accuracy of this assumption is open to question.

The same argument could be made against the storage-size measure; perhaps the accuracy of pattern recall did not adequately reflect the amount of information stored in memory. Unfortunately, most manipulations of storage size or processing effort are not directly measurable. By defining change in terms of the transition of neural systems from one state of excitation to another, however, the potential for direct measurement of this variable seems promising. Defining processing effort or storage size in terms of neural system excitation seems to ultimately involve the measurement of some form of change. For example, one could measure processing effort as reaction time or latency of evoked potentials, but this approach relies on counting changes in a physical monitor (i.e., the clock used to time these events); measuring storage size or processing effort as number or complexity of neural responses ultimately reduces to counting changes in electrical or metabolic/chemical states.

It can be argued that the validity of conclusions drawn from Experiments 2 through 5 is threatened by the fact that clock duration of test patterns was not varied. The concern is that in the forced-choice procedure subjects may have based their duration judgments on a nontemporal stimulus dimension because the relevant physical dimension was not varied. This implies that the dependent measure may not have accurately represented perceived duration.

Two related questions precipitate from this line of criticism. The first, and most fundamental, is whether it is possible to attend to time without attending to "nontemporal" information, and the second is whether it is likely that the present method caused subjects to ignore time in making their duration judgments.

The meaning of the first question is no doubt obfiscated by an obscure use of the terms "time" and "nontemporal." From the present perspective, it is assumed that when "time" is attended to, discrete physical changes are being experienced and ordered (i.e., "nontemporal" information is being monitored). Whether discrete change is the unit of psychological time or not, however, the idea that psycho- 
logical duration is related to the monitoring of physical events seems well founded as a direct extrapolation from the physical manifestation of clock timepassing events.

Ubiquitous empirical demonstration of the FDI shows clearly that nontemporal stimulus characteristics contribute to perceived duration; the simple fact that judged duration of empty time is a positive function of the interval's clock duration is evidence that covert (organismic) events contribute to duration estimates. Psychological time, like physical time, is therefore manifested as physical, "nontemporal" events.

Making this point essentially commits an answer to the second question-could the present method have lead subjects to base their duration judgments on something other than "time?" The line of reasoning followed in this paper leads to the conclusion that "time," as a dimension independent of other physical dimensions, cannot be apprehended; attending to "nontemporal" information is the only way to monitor duration."

The present explanation of duration experience parallels a previous model proposed by Thomas and Weaver (1975), according to which attention is shared between presented stimuli and "time," and perceived duration is constructed from the outcome of these two forms of processing. The change model proposed here specifies the objects of attention, those being sensory change (analogous to presented stimuli) and organismic change (analogous to what is referred to as "time").

It seems likely that, as attention proceeds from apperceptive changes, to changes in sensory or visceral organ systems, to physiological/chemical changes, the threshold value for change detection increases. It is also possible that some forms of change do not contribute to duration estimates at all, because they do not effect change in a superordinate mechanism. As a hypothetical example, eye movements, auditory pulses, light flashes, and doing math problems might each produce effects on perceived duration because they produce shifts of attention, which, in turn, might serve as the superordinate mechanism of time perception.

\section{CONCLUSION}

A model of psychological time based on change is parsimonious both because it does not implicitly require a timing mechanism independent of change and because it is consonant with our understanding of physical time. As noted by the physicist E. Mach: (1977) "It is utterly beyond our power to measure the changes of things by time. Quite the contrary, time is an abstraction, at which we arrive by means of the change of things"' (p. 273).
Man-made clocks log estimates of duration by detecting changes in physical states; the duration perception mechanism appears to log time by detecting changes in sensory states (which correspond to changes in physical states of the environment) and organismic states (which correspond to changes in the cognitive or physiological environment).

\section{REFERENCE NOTE}

1. Poynter, W. D. Processing time and apparent duration. Unpublished manuscript, 1980.

\section{REFERENCES}

AdAms, R. D. Intervening stimulus effects on category judgments of duration. Perception \& Psychophysics, 1977, 21, 527.534.

Avant, L. L., \& Lyman, P. J. Stimulus familiarity modifies perceived duration in prerecognition visual processing. Journal of Experimental Psychology: Human Perception and Performance, $1975,1,205-213$.

Avant, L. L., Lyman, P. J., \& Antes, J. R. Effects of stimulus familiarity on judged visual duration. Perception \& Psychophysics, 1975, 9, 327-334.

BLock, R. A. Memory and the experience of duration in retrospect. Memory \& Cognition, 1974, 2, 153-160.

BLоck, R. A. Remembered duration: Effects of event and sequence complexity. Memory \& Cognition, 1978, 6, 320-326.

BLock, R. A., \& REED, M. A. Remembered duration: Evidence for a contextual-change hypotheses. Journal of Experimental Psychology: Human Learning and Memory, 1978, 4, 656-665.

BuFfardi, L. Factors affecting the filled-duration illusion in the auditory, tactual, and visual modalities. Perception \& Psychophysics, 1971, 9, 404-406.

BURNSIDE, W. Judgment of short time intervals while performing mathematical tasks. Perception \& Psychophysics, 1971, 9, 404-406.

Craik, F.I.M., \& Tulving, E. Depth of processing and the retention of words in episodic memory. Journal of Experimental Psychology: General, 1975, 104, 268-294.

Fraisse, P. The psychology of time. New York: Harper and Row, 1963.

Francors, M. Contribution a l'étude du sens due temps: La temperature interne, comme facteur du variation de l'appreciation subjective des durées. L'Année Psychol., 1927, 186-204.

GARNER, W. R. Uncertainty and structure as psychological concepts. Huntington, N.Y: Wiley, 1975.

Glanzer, J., \& Clark, W. H. Accuracy of perceptual recall: An analysis of organization. Journal of Verbal Learning and Verbal Behavior, 1963, 1, 289-299.

Hicks, R. E., Miller, G. W., \& Kinsbourne, M. Prospective and retrospective judgments of time as a function of amount of information processed. American Journal of Psychology, 1976, 89, 719-730.

Hoagland, $H$. The physiological control of judgments of duration: Evidence for a chemical clock. Journal of General Psychology, 1933, 9, 267-287.

MAch, E. The science of mechanics. In R. Duncan \& M. WestonSmith (Eds.), The encyclopedia of ignorance. New York: Wallaby Books, 1977.

Mulligan, R. M., \& Schiffman, H. R. Temporal experience as a function of organization in memory. Bulletin of the Psychonomic Society, 1979, 14, 417-420.

Ornste in, R. E. On the experience of time. Middlesex, England: Penguin Books, 1969.

PoYNTE R, W. D. Human time perception and memory processes: The role of retrieval in duration estimates. Master's thesis, Arizona State University, 1979. 
Poynter, W. D. Duration judgment and the segmentation of experience. Memory \& Cognition, 1983, 11, 77-82.

Schiffman, H. R., \& Bовко, D. J. Effects of stimulus complexity on the perception of brief temporal intervals. Journal of Experimental Psychology, 1974, 103, 156-159.

Thomas, E.A.C., \& Brown, I., JR. Time perception and the filled-duration illusion. Perception \& Psychophysics, 1974, 16, 449-458.

Thomas, E.A.C., \& Weaver, W. B. Cognitive processing and time perception. Perception \& Psychophysics, 1975, 17, 363367.

Vroon, P. A. Effects of presented and processed information on duration experience. Acta Psychologica, 1970, 34, 115-121.

\section{NOTES}

1. This categorization of time-perception models follows from Block and Reed (1978).

2. $\mathrm{FOC}$ for filler $=2.8$.
3. Same filler as for Experiment 2, FOC $=4.4$.

4. Same filler as for Experiment 3, FOC $=3.3$.

5 . Filler 1 had six flashes in the same spatial locations as the experimental patterns, with ISIs $=.5, .3, .2, .4,0, .1 ;$ FOC $=5.2$. Filler 2 consisted of six sequential flashes in one location with ISIs of $.1, .1, .1, .1, .1,1.0 ;$ FOC $=3.1$.

6. This by no means totally discounts the possibility that stimulus dimensions that did not contribute to the experience of duration were used to make a decision in the force-choice task; given the explicit directions to base judgments on perceived duration (and make an arbitrary choice otherwise), there is no compelling evidence to believe they did so. Regardless of the timeestimation task used (magnitude estimation, forced choice with variable durations, reproduction, etc.), however, there is always some possibility that information irrelevant to the duration experience has, to some degree, contaminated the time judgment.

(Manuscript received October 18, 1982; revision accepted for publication February 14, 1983.) 